\section{ken wyle}

Ice sheet
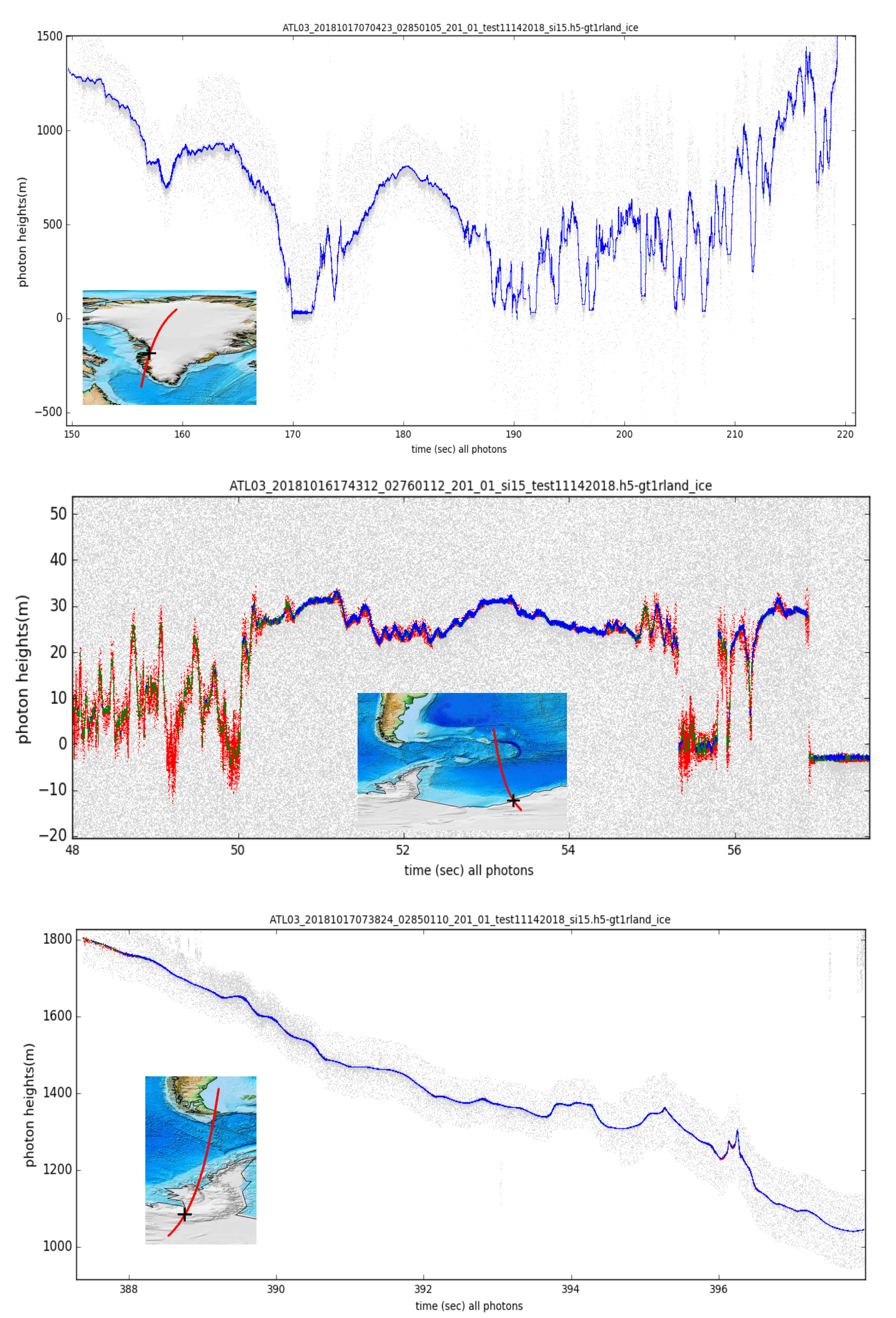

Geolocated Photon Data Product

Anita C. Brenner ${ }^{1}$, Thomas Neumann², Jeffrey E. Lee ${ }^{3}$ Actual Photon Classification on ATL03 Sea ice
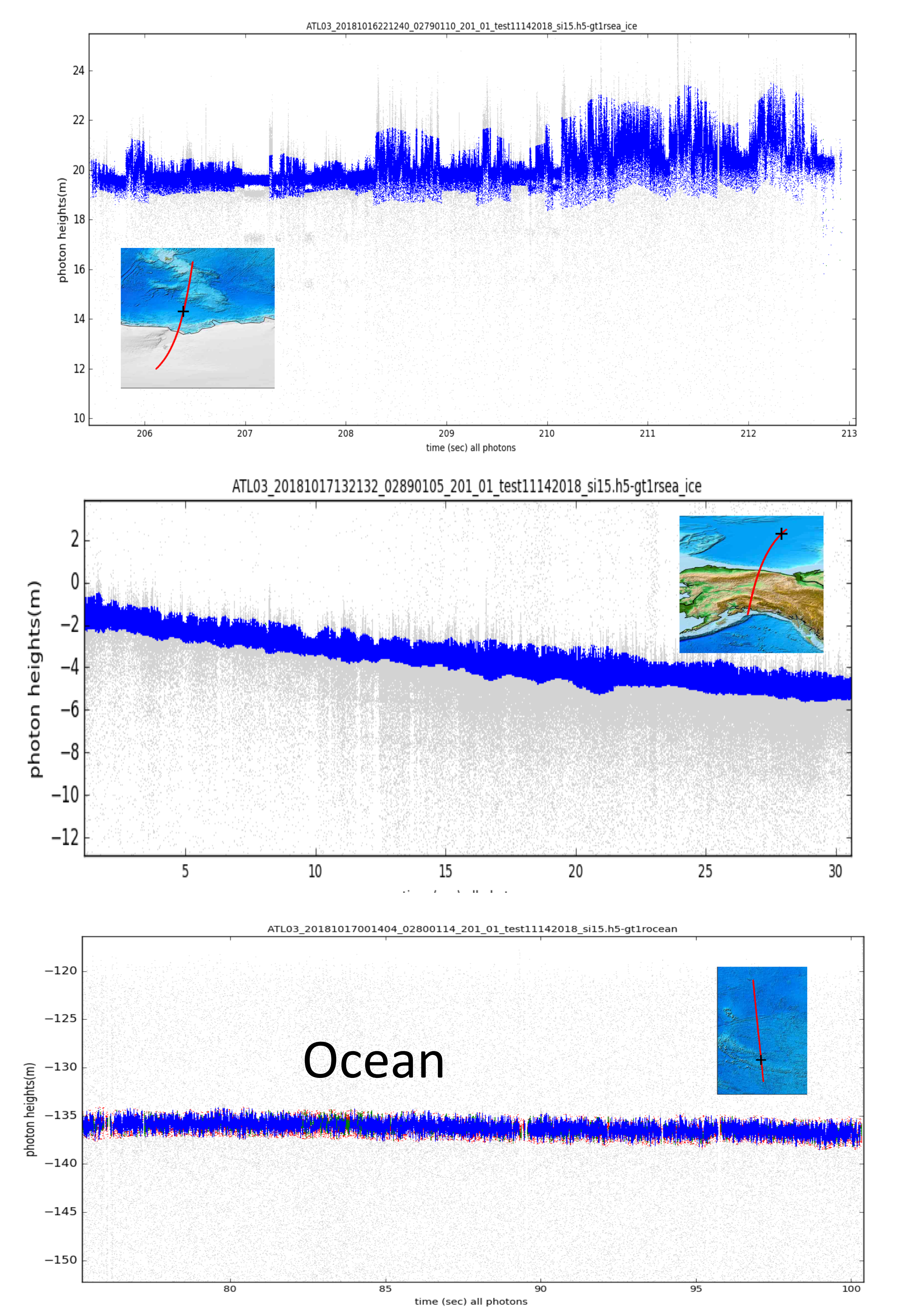

Land
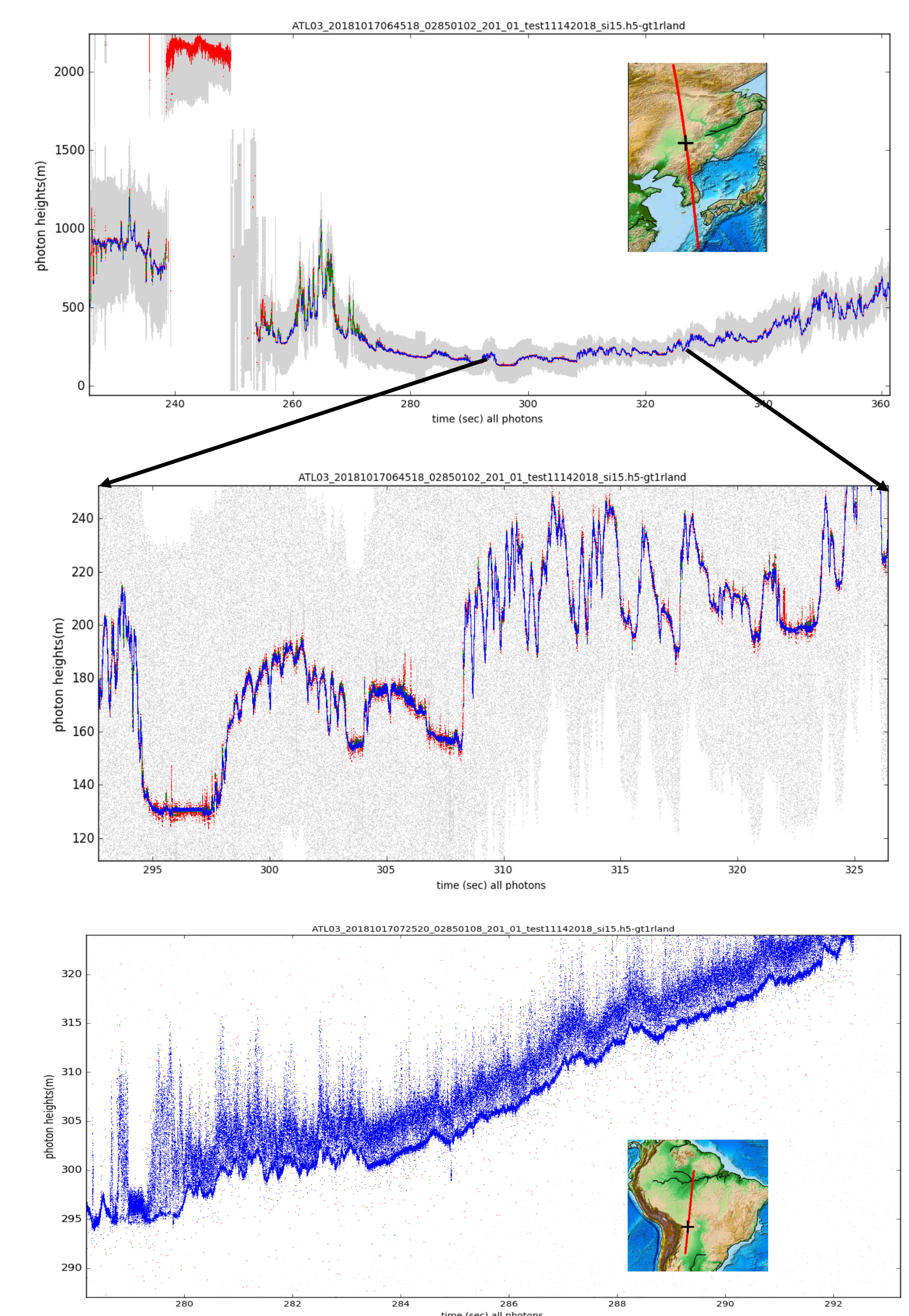

Land

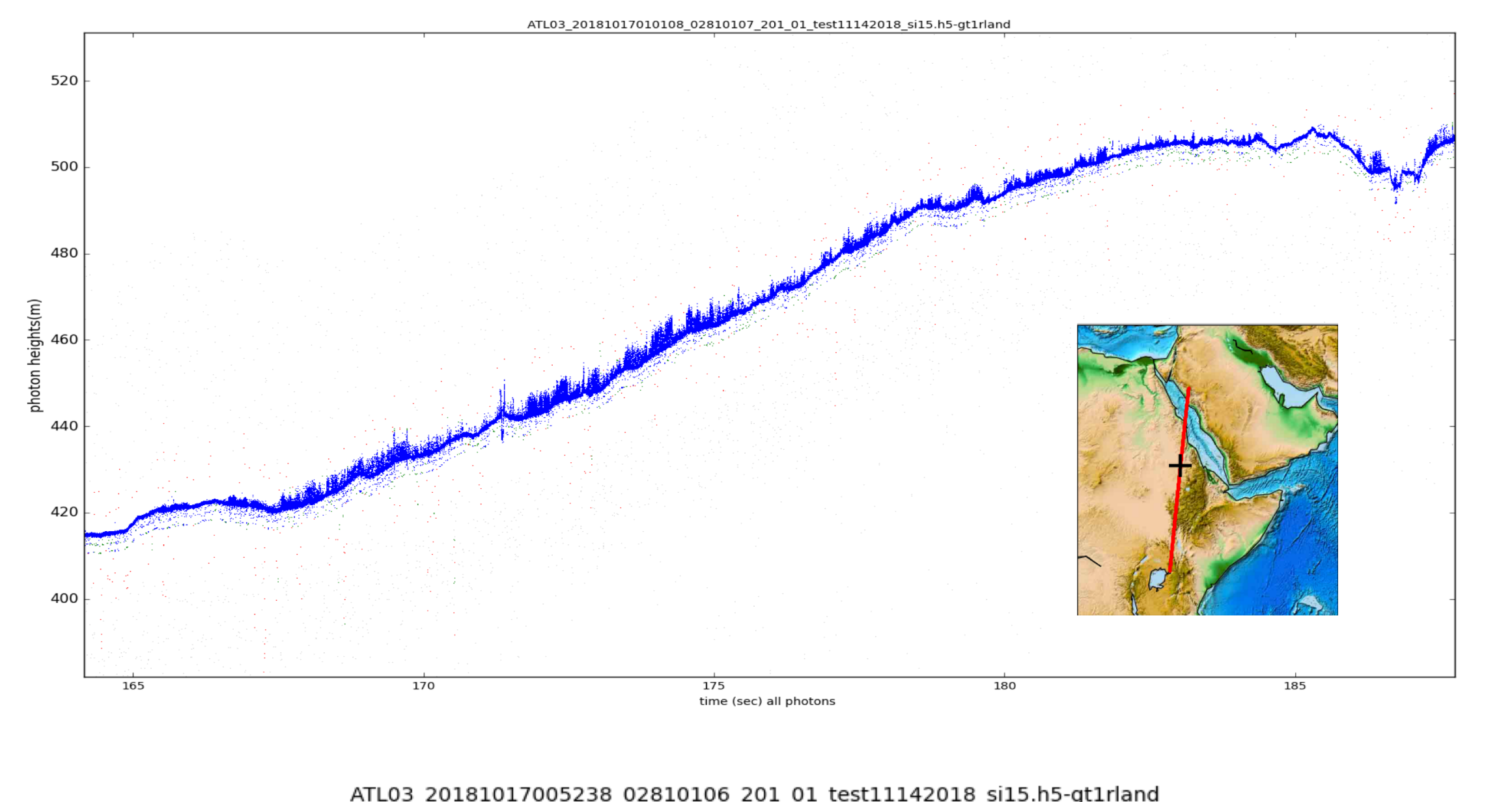

Land ând inland water

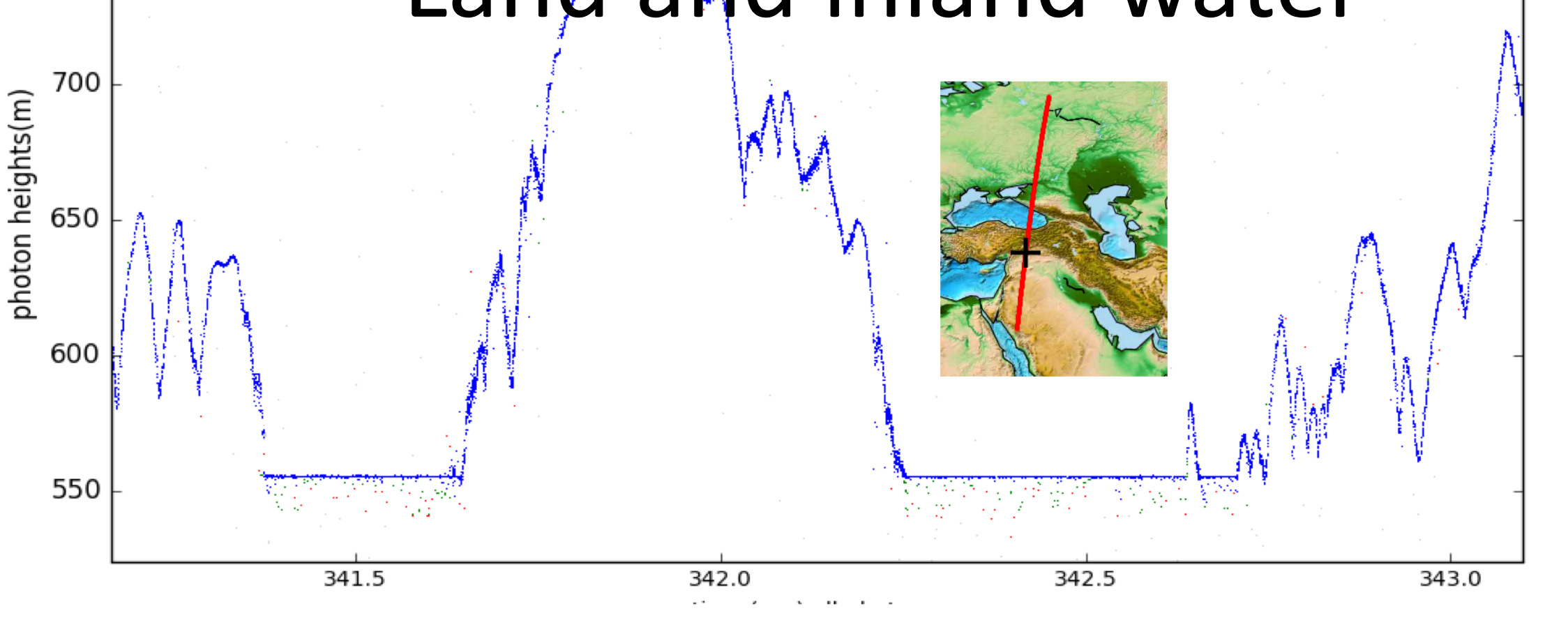

Inland water

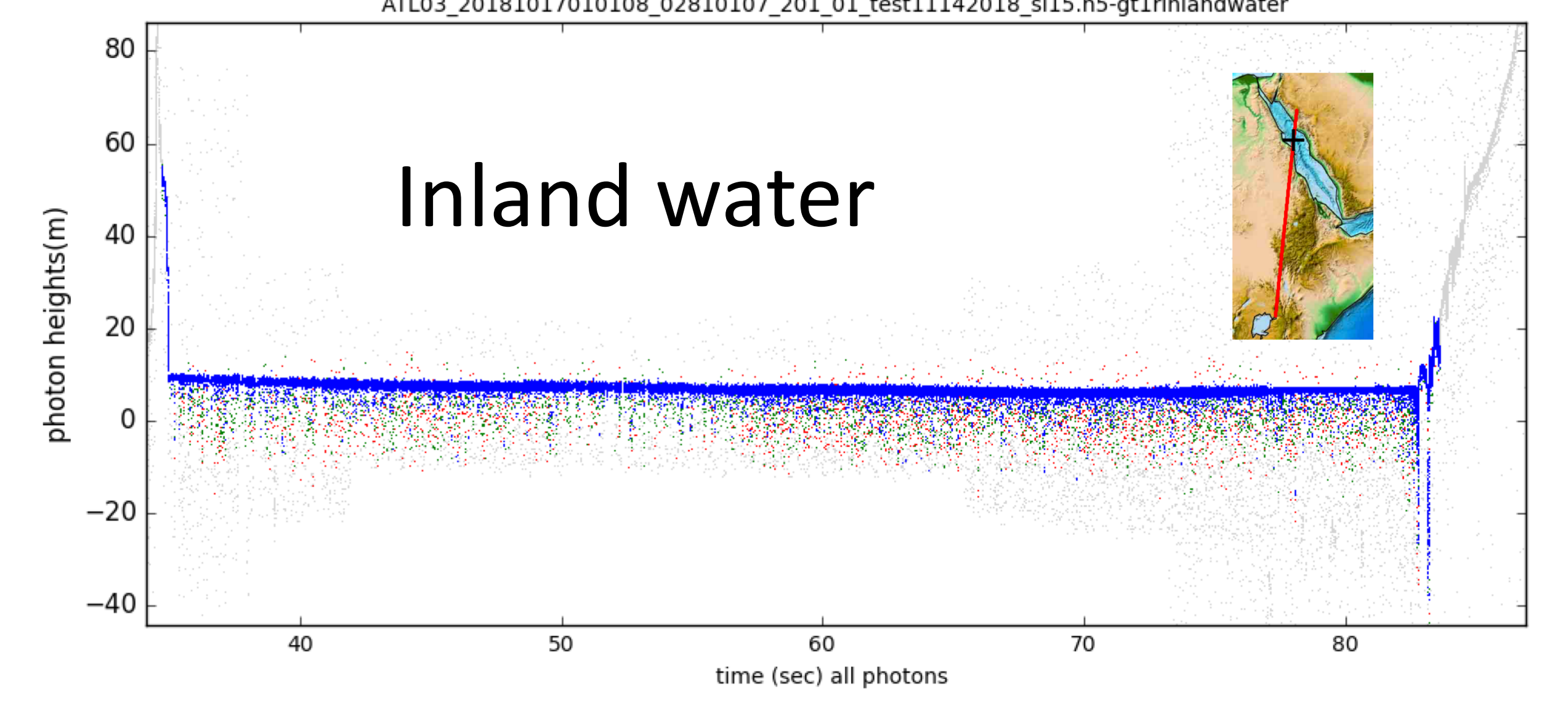

Photon classification Key

Medium confidence signal _L Low confidence signal $\quad$ Non-signal (noise)

\title{
Photon Classification Algorithm
}

Problem - telemetered photon events (spanning $30 \mathrm{~m}$ to $2000 \mathrm{~m}$ in heights) on ATL03 contain both signal and background photon events.

Solution - a photon classification algorithm to provide an initial discrimination between signal and background photon events.

Goal - identify all signal photon events while minimizing false positives
A: Determine background photon rate
1. Use atmospheric histogram: sum of 400 laser shots in $30 \mathrm{~m}$ height bins spanning $\sim 14 \mathrm{~km}$ in height
a) Remove bins containing signal using $n *$ sigma editing
b) Calculate mean, $\mu_{\mathrm{bg}}$, and standard deviation, $\sigma_{\mathrm{bg}}$, of remaining bins

B: Calculate a signal threshold: $\operatorname{Sig}_{\mathrm{th}}=\mu_{\mathrm{bg}}+\mathrm{e}_{\mathrm{m}} \times \sigma_{\mathrm{bg}}$

C: Histogram the ellipsoidal height of the telemetered photons over time period, $\mathbf{d t}$, using height bins of size $\mathbf{d z}$.

1. Scale sig th to $d t$ and $d z$

2. Identify signal bins as those that exceed sig th $_{\text {th }}$

3. Throw out signal bins where the number of photons is $<r x$ maximum number of photons in one histogram bin

4. Add bins on each side of the signal bins until find two bins where the number of photons $<\mu_{\mathrm{bg}}$

5. If no signal is found increment $\mathbf{d t}$ and $\mathbf{d z}$ and iterate until maximum values of $\mathrm{dt}$ and $\mathrm{dz}$ reached.
D: Identify additional signal missed by ellipsoidal height histogramming

1. Strong beams over land or land ice histogram the height relative to the surface slope as defined by signal identified in c. Perform steps as in c to identify additional signal.

2. Weak beams over all surfaces histogram the height relative to the surface slope defined by signal photon events corresponding to the strong beam in the pair after adjusting for the fore/aft offset between the weak and strong beams. Perform steps as in c to identify additional signal.

E: Assign a confidence level to each photon event for each surface type:

1. Identified signal

a) 4: High-confidence signal (SNR $\geq 100)$

b) 3: Medium-confidence signal $(100>\operatorname{SNR} \geq 40)$

c) 2: Low-confidence signal $(40>S N R \geq 3)$

2. Non-signal photon confidence levels:

a) 1: within $+/-10 \mathrm{~m}$ of mean surface calculated from the signal-identified photons

b) 0: probable background photon event

c) -1: not assessed for that surface type

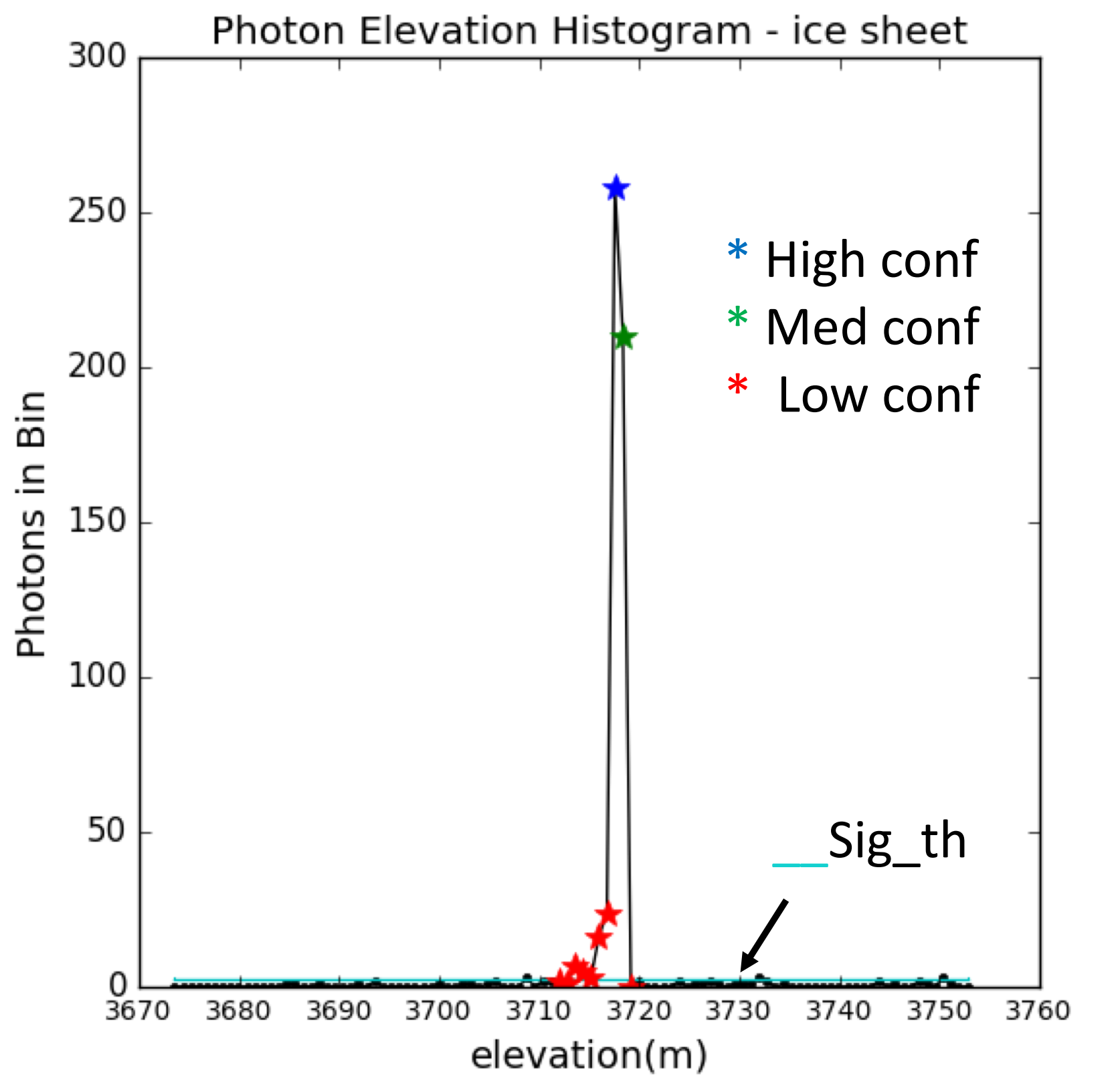

signal_conf_ph:

Five columns: land, ocean, sea ice, land ice, inland water

$N$ rows: corresponds to number of photon events 